\title{
Increased risk of community-acquired pneumonia in COPD patients with comorbid cardiovascular disease
}

This article was published in the following Dove Press journal:

International Journal of COPD

5 December 2016

Number of times this article has been viewed

\author{
Sheng-Hao Lin ${ }^{1,2}$ \\ Diahn-Warng Perng ${ }^{3,4}$ \\ Ching-Pei Chen ${ }^{5,6}$ \\ Woei-Horng Chai' \\ Chin-Shui Yeh' \\ Chew-Teng Kor ${ }^{7}$ \\ Shih-Lung Cheng 8,9 \\ Jeremy JW Chen ${ }^{2, *}$ \\ Ching-Hsiung Lin ${ }^{1,10,11, *}$
}

'Department of Internal Medicine, Division of Chest Medicine, Changhua Christian

Hospital, Changhua, ${ }^{2}$ Institute of Biomedical Sciences, National Chung Hsing University,

Taichung, ${ }^{3}$ Department of Chest Medicine,

Taipei Veterans General Hospital, Taipei,

${ }^{4} \mathrm{~S}$ chool of Medicine, National Yang-

Ming University, Taipei, ${ }^{5}$ Department of

Internal Medicine, Division of Cardiology,

Changhua Christian Hospital, ${ }^{6}$ Department

of Beauty Science and Graduate Institute

of Beauty Science Technology, Chien-Kuo

Technology University, ${ }^{7}$ Department

of Internal Medicine, Internal Medicine

Research Center, Changhua Christian

Hospital, Changhua, ${ }^{8}$ Department of

Internal Medicine, Far Eastern Memorial

Hospital, Taipei, ${ }^{9}$ Department of Chemical

Engineering and Materials Science, Yuan

Ze University, Zhongli City, Taoyuan,

${ }^{10}$ Department of Respiratory Care,

College of Health Sciences, Chang Jung

Christian University, Tainan, "School of

Medicine, Chung Shan Medical University,

Taichung, Taiwan

*These authors contributed equally to this work

Correspondence: Ching-Hsiung Lin

Department of Internal Medicine, Division of

Chest Medicine, Changhua Christian Hospital,

I 35 Nanxiao St, Changhua City, Changhua

County 500, Taiwan

Tel +886472385951039

Fax +88647232942

Email teddy@cch.org.tw
Background and objective: COPD patients with community-acquired pneumonia (CAP) have worse clinical outcomes, as compared to those without COPD. Cardiovascular disease (CVD) is a common comorbidity for COPD patients. Whether COPD with comorbid CVD will increase the risk of CAP is not well investigated. The incidence and factors associated with CAP in COPD patients with and without CVD were analyzed.

Methods: The medical records of patients with newly diagnosed COPD between 2007 and 2010 were reviewed. The patients' characteristics, medical history of CVD, occurrence of CAP, and type of medication were recorded. Kaplan-Meier curves were used to assess the differences in cumulative incidence of CAP. Cox's proportional hazards regression model was used to determine the adjusted hazard ratios with $95 \%$ confidence intervals in relation to factors associated with CAP in COPD patients with and without CVD.

Results: Among 2,440 patients, 475 patients (19.5\%) developed CAP during the follow-up period. COPD patients who developed CAP were significantly older, had lower forced expiratory volume in 1 second, frequent severe exacerbation and comorbid CVD, as well as received inhaled corticosteroid (ICS)-containing therapy than those without CAP. The cumulative incidence of CAP was higher in COPD patients with CVD compared to those without CVD. Patients who received ICS-containing therapy had significantly increased risk of developing CAP compared to those who did not.

Conclusion: For patients with COPD, comorbid CVD is an independent risk factor for developing CAP. ICS-containing therapy may increase the risk of CAP among COPD patients.

Keywords: chronic obstructive pulmonary disease, COPD, community-acquired pneumonia, cardiovascular disease, inhaled corticosteroids, CAP, CVD

\section{Introduction}

COPD is the leading cause of death for both males and females in the US and is projected to rise in ranking by $2020 .{ }^{1}$ According to data from the National Center for Health Statistics of the Centers for Disease Control and Prevention, COPD became the third leading cause of death by 2008. ${ }^{1,2}$ Like other chronic diseases, COPD is usually related to pneumonia ${ }^{3}$ and is the most common fundamental clinical condition for patients who need intensive care due to severe community-acquired pneumonia (CAP). ${ }^{4}$ Compared to patients without COPD, CAP patients with COPD are likely to have more severe pneumonia, increased number of hospital admissions, and worse outcome. ${ }^{5-8}$

The risk factors for acquiring CAP include increasing age and comorbid illnesses such as neoplasia, liver disease, renal disease, cardiac failure, and altered mental 
status. ${ }^{9}$ In Europe, one review article showed that chronic cardiovascular disease (CVD) increases the risk of CAP in adults, even up to threefold. Other studies also showed that chronic heart disease and heart failure are the risk factors of CAP ${ }^{10}$ Another cohort study of patients with an episode of CAP that was conducted to predict mortality after discharge showed that comorbid cerebrovascular disease and CVD, altered mental state, anemia, hyperglycemia, and increasing age were independent predictors of mortality. ${ }^{11}$ Furthermore, incident cardiac complications such as myocardial infarction and new or worsening arrhythmias/heart failure are common in patients with CAP and are related to increased short-term mortality. ${ }^{12}$

Inhaled corticosteroids (ICSs) is one of the recommended pharmacotherapies for COPD by the Global Initiative for Chronic Obstructive Lung Disease (GOLD). ${ }^{13}$ However, many studies have shown that ICSs may increase the risk of pneumonia and pneumonia-related hospitalization in COPD patients. ${ }^{14-16}$ The underlying mechanism has not been understood clearly, although the anti-inflammatory effects and local immune suppression of the airways may be involved. ${ }^{17-19}$
Compared to heart failure, limited data are available regarding the impact of CVD on the occurrence of CAP in COPD patients. This retrospective study aimed to investigate the impact of comorbid CVD in hospitalized COPD patients with CAP. We hypothesized that COPD patients with CVD may have increased risk of developing CAP. The use of ICSs was also assessed to examine the risk of developing CAP in COPD patients.

\section{Materials and methods Patients and study design}

COPD was diagnosed according to the GOLD guidelines. ${ }^{13}$ The medical records of patients with newly diagnosed COPD (ICD-9: 496) between January 1, 2007 and December 31, 2010 were reviewed. Patients who had no pulmonary function data or who had history of asthma were excluded. A total of 2,440 patients with COPD were finally enrolled (Figure 1). The patients' characteristics, medical history of CVD, occurrence of CAP, and type of medication were recorded. The enrolled patients had been followed for at least 3 years. The median follow-up periods (interquartile range) for patients without

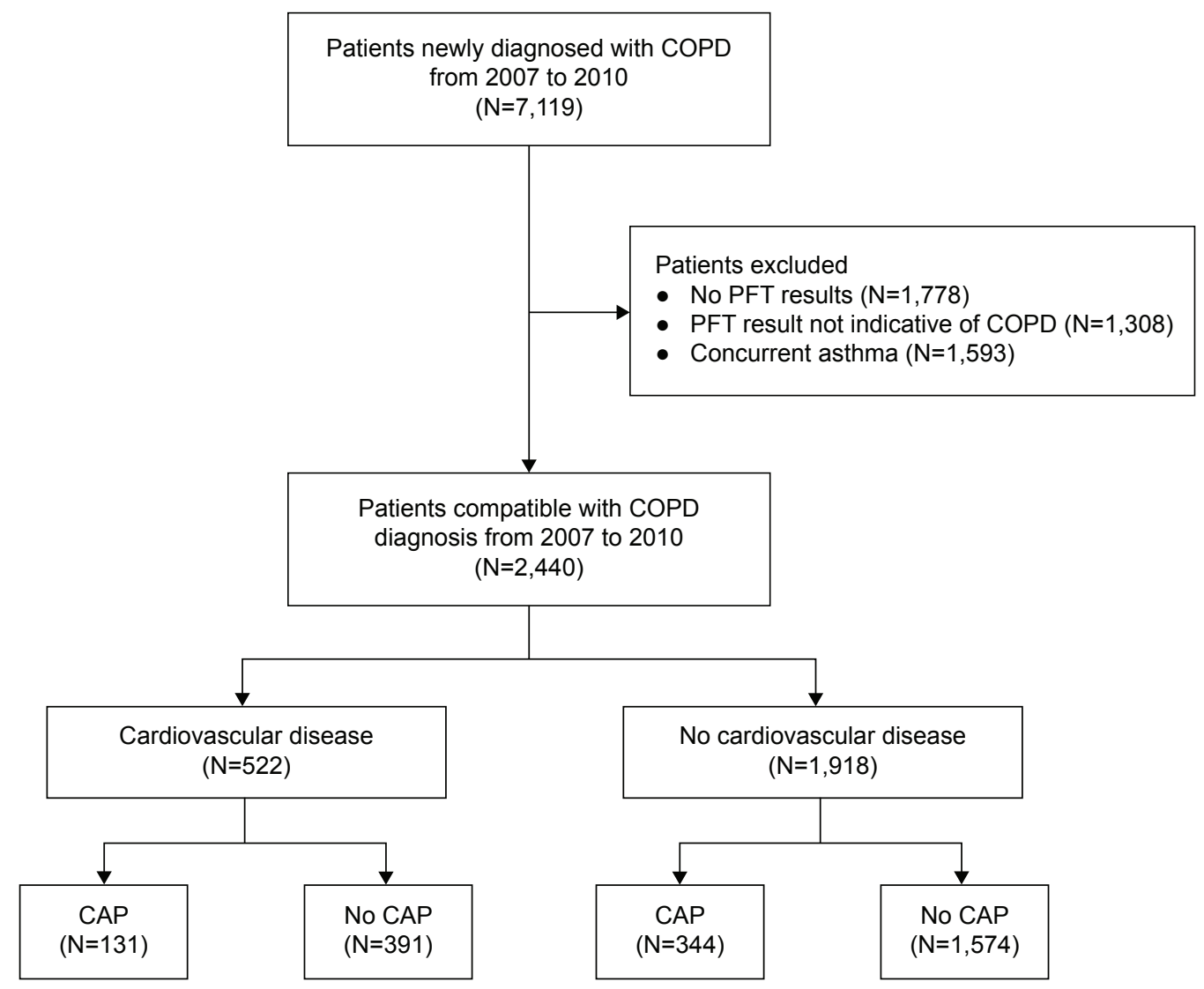

Figure I Flowchart of study profile.

Abbreviations: CAP, community-acquired pneumonia; PFT, pulmonary function test. 
ICS-containing therapy and CVD, patients without ICScontaining therapy but with cardiovascular disease (CVD), patients with ICS-containing therapy but without CVD, and patients with ICS-containing therapy and CVD were 54.8, $54.8,57.1$, and 58.5 months, respectively. There was no statistically significant difference in the follow-up period among groups. All medical records were deidentified prior to analysis. The database was analyzed anonymously by using encrypted personal identification number. The Institutional Review Board of Changhua Christian Hospital approved the study and protocol (CCH-IRB-121218) and deemed patient consent was not required as this was a retrospective study using de-identified patient data.

\section{Variables and definitions}

COPD was defined as post-bronchodilator forced expiratory volume in 1 second $\left(\mathrm{FEV}_{1}\right)$ to forced vital capacity (FVC) ratio of less than 0.70 , while COPD severity was classified according to the GOLD staging. ${ }^{13}$ A CAP diagnosis was reviewed by the research team based on confirmatory clinical findings, including new chest radiographic infiltrates, presentation of cough, sputum production, fever $>37.8^{\circ} \mathrm{C}$, dyspnea, altered mental status, pleuritic chest pain, and/ or leukocytosis $>12,000 / \mathrm{mm}^{3}$, and isolation of pathogens from respiratory samples. The presence of CVD was diagnosed by symptoms of patients, combined with positive coronary artery angiography, myocardial perfusion scan, multidetector computed tomography of cardiac angiography, echocardiogram, or treadmill stress test performed at the cardiovascular outpatient clinic. Patients who had only mild symptoms, but significant CVD on angiography or computed tomography were also included. The presence of diabetes mellitus (DM) was diagnosed by either fasting glucose level $>126 \mathrm{mg} / \mathrm{dL}$ or Glycated hemoglobin A1C $(\mathrm{HbA} 1 \mathrm{c})>6.5 \%$. Patients with DM were regularly treated with an oral hypoglycemic agent or insulin. Severe exacerbation of COPD was defined as emergency room visit or hospitalization.

\section{Statistical analysis}

Due to non-normal distributions, results for continuous variables are given as medians with interquartile ranges (25th-75th percentiles). Results for categorical variables are presented in frequencies and percentages. Comparisons of demographic and clinical characteristics among patients who did and did not develop CAP were performed by Wilcoxon rank sum tests for continuous variables and by chi-square tests for categorical variables.
The time between COPD diagnosis and first CAP occurrence was considered as the time to pneumonia. Death without CAP occurrence was treated as a competing risk event since death may preclude CAP occurrence, thereby censoring the estimation of CAP incidence.

To investigate the factors related to CAP occurrence, Cox proportional hazards regression with competing risk analysis was used to calculate the crude hazard ratios and adjusted hazard ratios (aHRs) and the 95\% confidence intervals (CIs) according to the approach of Fine and Gray. ${ }^{20}$ The multivariate analysis was adjusted for ICSs and CVD group, gender, age, body mass index (BMI), $\mathrm{FEV}_{1} \%$ predicted, exacerbation frequency, DM, comorbid pulmonary disease, and cerebral vascular disease. The cumulative incidence curve was plotted according to this revision model. Cumulative incidence curves for estimating the probability of progression to pneumonia over time were generated, and Gray's test was used to test for differences between the study groups. Calculations of cumulative incidences and all competing risk analyses were conducted using the "cmprsk" package (version 3.1.2; https://cran.r-project.org/web/packages/cmprsk/index.html), while the "survival" package (https://cran.r-project.org/web/ packages/survival/index.html) was used to generate cumulative incidence plots. A two-tailed $P<0.05$ was considered statistically significant.

\section{Results}

Of the 2,440 patients included in the analysis, 522 patients (21.4\%) had CVD. By the end of the study period, 475 patients (19.5\%) had developed CAP during the follow-up period. Based on the patient characteristics, patients who developed CAP were significantly older, had lower $\mathrm{FEV}_{1}$, comorbid DM, more frequent severe exacerbation and comorbid CVD, as well as had received ICS-containing therapy compared to those without CAP (Table 1). By using multivariate regression analysis, increased age (aHR: 1.03; 95\% CI: 1.02-1.04), $\mathrm{FEV}_{1} \%$ predicted (aHR: 1.0; 95\% CI: 0.99-1.0), more frequent severe exacerbation (aHR: 1.10; 95\% CI: 1.07-1.12), ICS-containing therapy (aHR: 1.26; 95\% CI: 1.03-1.54), the presence of CVD (aHR: 1.28; 95\% CI: 1.05-1.57), DM (aHR: 1.54; 95\% CI: 1.21-1.95), and comorbid pulmonary disease (inclusive of bronchiectasis, history of tuberculosis and lung cancer) (aHR: 1.91; 95\% CI: 1.52-2.40), were found to be associated with increased risk of developing CAP (Table 2).

Among the 2,440 COPD patients, intensive care unit admission was $11.45 \%$ in patients with CVD and $15.41 \%$ in patients without CVD. The $P$-value was not significant. 


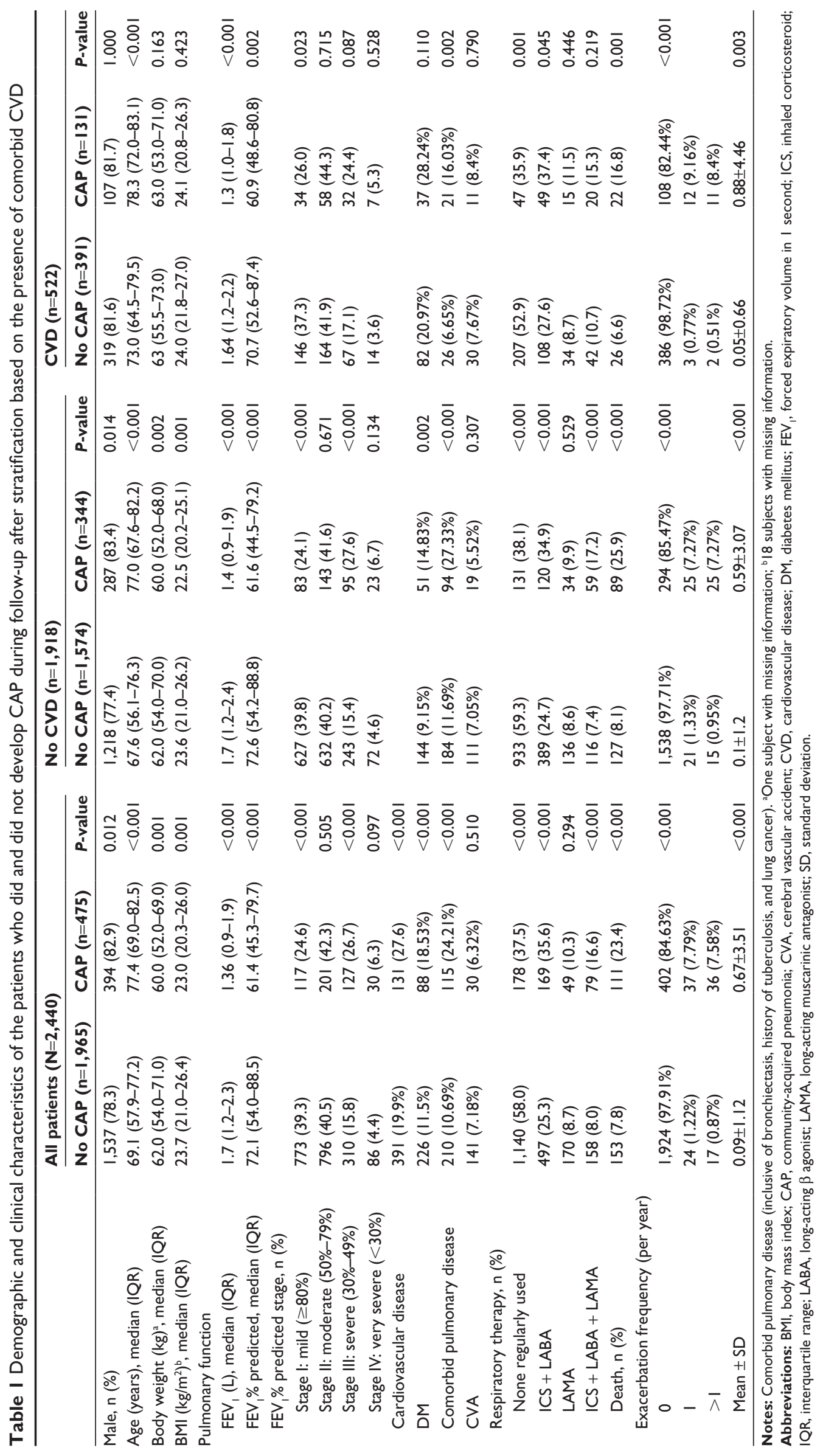




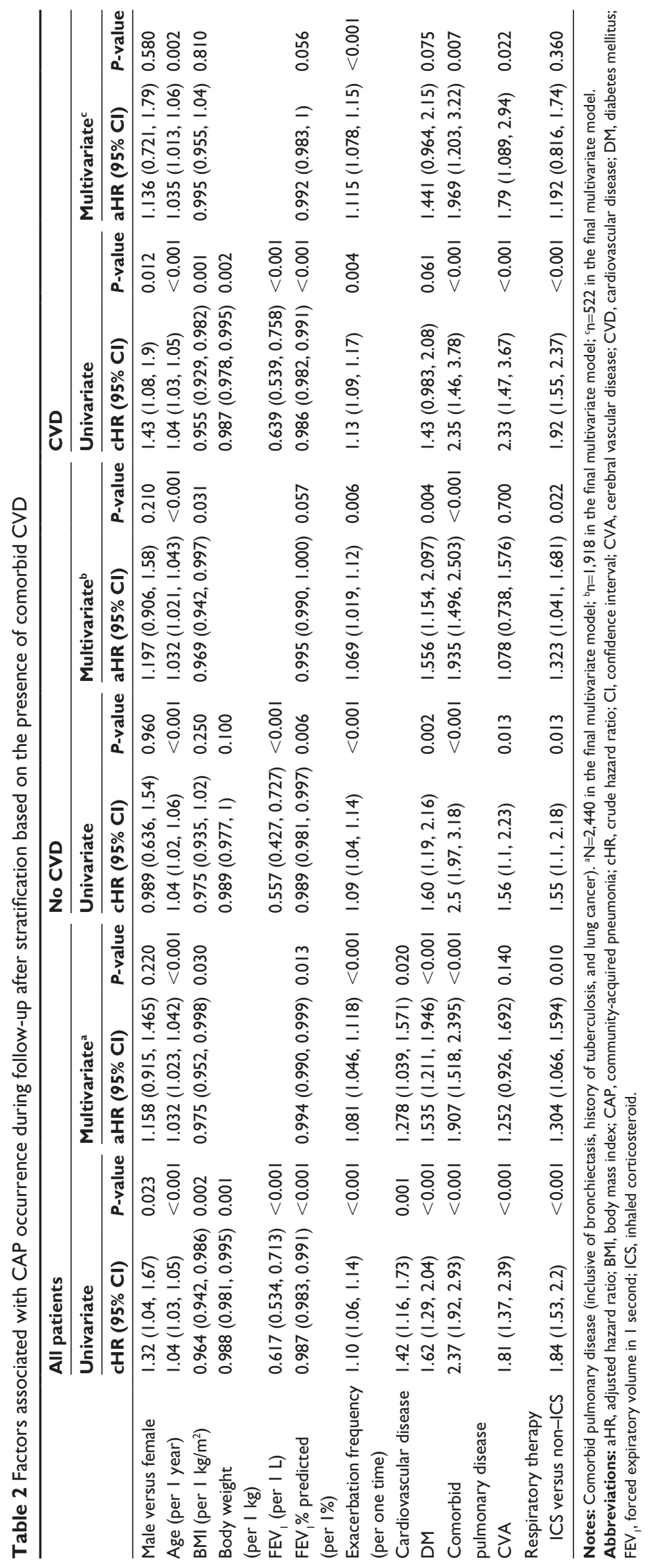




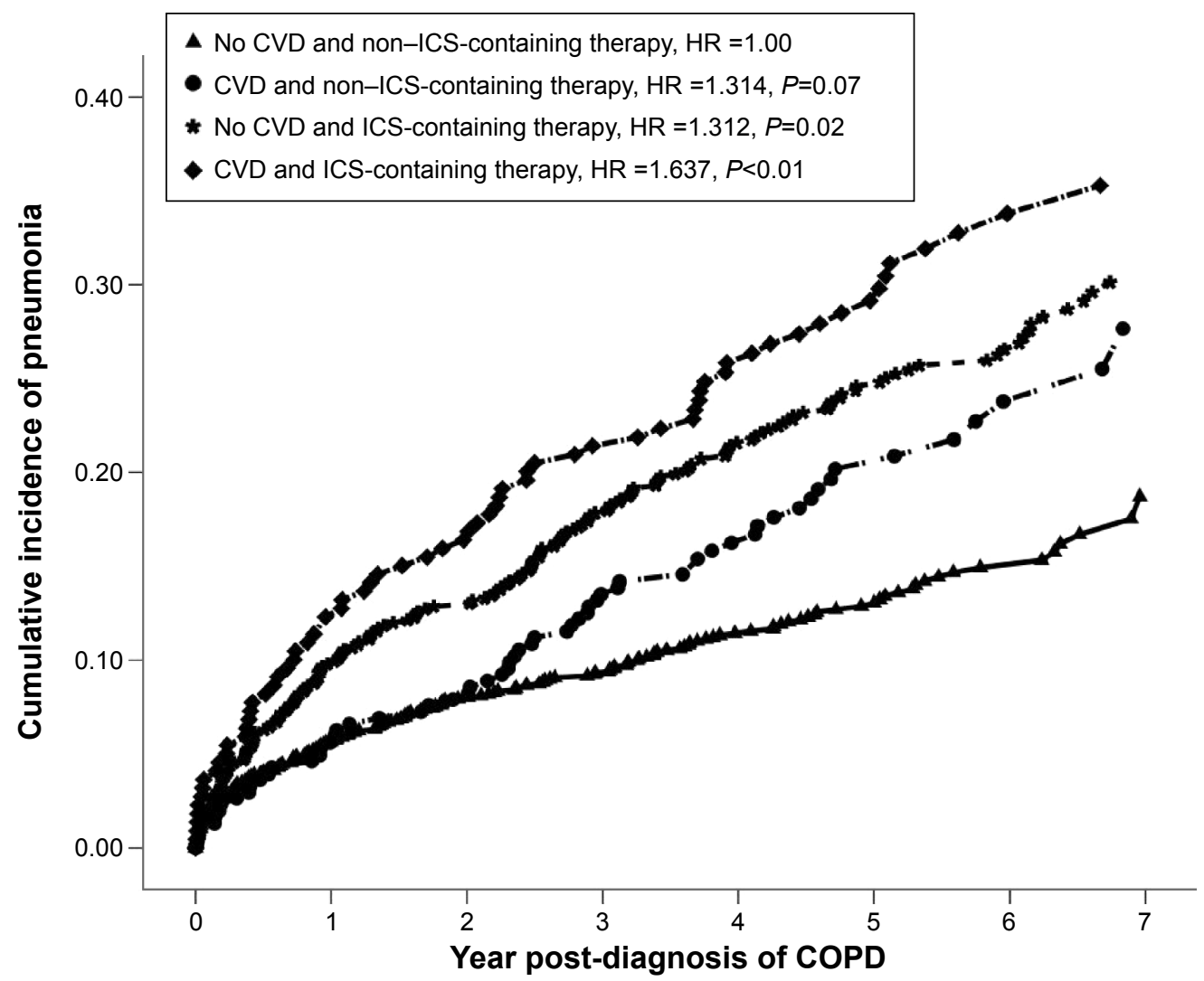

Figure 2 Kaplan-Meier curve for pneumonia-free survival of COPD patients stratified by comorbid CVD and ICS-containing therapy (N=2,440).

Notes: These groups include patients without CVD with ICS-containing therapy, patients without CVD with non-ICS-containing therapy, patients with CVD with ICScontaining therapy, patients with CVD with non-ICS-containing therapy.

Abbreviations: CVD, cardiovascular disease; HR, hazard ratio; ICS, inhaled corticosteroid.

Therefore, CVDs have no effects on the clinical outcome of CAP in terms of intensive care unit admission (data not shown).

Cumulative incidence curves for estimating the probability of progression into pneumonia over time are presented in Figure 2. Significant differences were found between the four study groups $(P<0.01$, by Gray's test). After 2 years follow-up, the cumulative incidence of pneumonia was higher in the group of COPD patients with CVD than in those without CVD. Of particular interest, patients with comorbid CVD who received ICS-containing therapy had significantly increased risk of developing CAP than those who did not receive ICS-containing therapy or those who only had comorbid CVD (Figure 2) $(P<0.01$, by log-rank test).

In subgroup analysis based on the absence of CVD, after adjusting for other potential confounders, age, lower $\mathrm{FEV}_{1} \%$ predicted, severe exacerbation frequency, and ICS-containing therapy were found to be the potential risk factors of CAP (Table 2). For patients with comorbid CVD, multivariate analysis revealed that age and frequent severe exacerbation increased the risk of developing CAP (Table 2). Although all patients with comorbid DM had higher incidence of CAP, multivariate analysis showed no significant difference in the incidence of CAP in the subgroup of patients with comorbid CVD (CAP $28.2 \%$ vs without comorbid CVD $21 \%, P=0.11$ ) (Table 1 ).

\section{Discussion}

CVDs are the most common comorbid diseases of COPD. ${ }^{18,19,21}$ Pre-existing CVD may increase long-term mortality in elderly COPD patients with pneumonia. ${ }^{21}$ A 5-year prospective study showed that CVD was the most common risk factor of long-term mortality after hospitalization for CAP ${ }^{22}$ In this study, the presence of CVD in COPD patients was found to be an independent risk factor for developing CAP. COPD patients who developed CAP were significantly older, had lower $\mathrm{FEV}_{1}$, more frequent severe exacerbation and comorbid CVD, and received ICS-containing therapy compared to those without CAP.

Cardiac comorbidities are highly prevalent in COPD patients. The prevalence of CVD in COPD ranges from 13\% to $68 \% .{ }^{23}$ One review article mentioned that ischemic heart disease (IHD) was the most frequent cardiac comorbidity in 
COPD patients. The risk of developing IHD, and particularly, acute coronary syndrome, is significantly higher in COPD patients, as compared to the general population. COPD is also frequent in IHD patients, ranging in incidence from $4 \%$ to $18 \%{ }^{24}$ The prevalence rate of CVD in COPD patients is $21.4 \%$ in this study, and is higher than those in other reports due to the different criteria used to define CVD. Patients with either COPD or CVD may share common risk factors, aging process, disability, and systemic inflammation pathways. Several studies have shown that COPD may increase the risk of CVD independently, and an increased risk of mortality trend was also found in COPD patients with comorbid CVD. ${ }^{21,25,26}$ Another study also reported similar results in the CVD patients with pneumonia. ${ }^{27,28}$ In a review article about the risk factors of pneumonia, chronic CVD increased the risk of CAP up to threefold, with crude odds ratios ranging from 1.4 to 3.2. Some studies supported an association between chronic heart disease or heart failure and the risk of CAP. ${ }^{10}$ Compared to heart failure, limited data are available regarding the impact of CVD on the occurrence of CAP in COPD patients. Therefore, a better understanding about the increased risk of CAP in COPD patients with CVD may help clinicians understand how to improve the clinical outcomes of the patients.

The overall incidence of CAP in COPD patients was $19.5 \%$ in this study, which is higher than the incidence ( $8 \%$ ) reported by Müllerova et al. ${ }^{29}$ The difference between our study and Müllerova et al may be attributed to higher CVD comorbidity and COPD severity according to baseline patient characteristics in our study. In addition to CVD and COPD, increased age ( $\geq 65$ years) has been considered as an independent risk factor for severe CAP. ${ }^{30}$ Towards a Revolution in COPD Health study, which was a large prospective and randomized study performed for a period of 3 years, reported that both increased age ( $\geq 55$ years) and reduced BMI $\left(<25 \mathrm{~kg} / \mathrm{m}^{2}\right)$ were independent risk factors for developing CAP in COPD patients. ${ }^{31}$ Lange et al also found that reduced BMI $\left(<20 \mathrm{~kg} / \mathrm{m}^{2}\right)$ was a contributing factor of death due to pneumonia. ${ }^{32}$ The reason might be that elderly people are likely to have deteriorated functional status, increased disease severity, and coexisting medical illnesses, ${ }^{33}$ and that BMI may have strong association with immune response. ${ }^{34}$

It was observed in this study that ICS-containing therapy was associated with a higher risk of pneumonia. This finding is supported by growing evidence which implies that ICSs use is a risk factor for pneumonia in COPD patients, because it can increase the relative risk of pneumonia by $34 \%-60 \% .{ }^{15,16}$ However, there is no significantly increased risk of death for COPD patients with ICSs use, as reported by those meta-analyses. Recently, a retrospective pairwise cohort study in Sweden reported the increased risk of pneumonia and pneumonia-related events in COPD patients who received fixed combinations of ICS/long-acting $\beta_{2}$ agonist with an intraclass difference. ${ }^{35}$ Furthermore, for patients with comorbid CVD, multivariate analysis revealed that ICS-containing therapy increased the risk of developing CAP. Future studies should investigate whether specific subsets of patients with COPD (with regard to underlying comorbidity) can benefit from ICS therapy.

The limitations in this study should be addressed. Firstly, it was an observational retrospective analysis. The causes of death of the COPD patients were not examined in detail. We cannot determine the interrelation between CVD, CAP, and COPD regarding their impact on mortality. Secondly, the dosage, class, and duration of ICSs use for the COPD patients were not analyzed thoroughly. Thirdly, the mechanism underlying increased risk of CVD is not clear at this stage. Future studies in this regard are warranted.

\section{Conclusion}

This study revealed that COPD patients with comorbid CVD had increased risk of CAP. Patients with comorbid CVD who received ICS-containing therapy had significantly increased risk of developing CAP compared to those who did not receive ICS-containing therapy or those who only had comorbid CVD.

\section{Acknowledgments}

The authors thank the International Medical Humanities and Health Sciences Academy of Changhua Christian Hospital for their assistance.

\section{Disclosure}

The authors report no conflicts of interest in this work.

\section{References}

1. Heron M. Deaths: leading causes for 2010. National vital statistics reports Centers for Disease Control and Prevention, National Center for Health Statistics, National Vital Statistics System. 2013;62:1-97.

2. Chronic obstructive pulmonary disease. In: Services UDoHaH, (ed.) Washington, DC: USDHHS; Data fact sheet; 2003.

3. Mannino DM, Davis KJ, Kiri VA. Chronic obstructive pulmonary disease and hospitalizations for pneumonia in a US cohort. Respir Med. 2009; 103(2):224-229.

4. Torres A, Serra-Batlles J, Ferrer A, et al. Severe community-acquired pneumonia. Epidemiology and prognostic factors. Am Rev Respir Dis. 1991;144(2):312-318.

5. Chen Y, Stewart P, Dales R, Johansen H, Bryan S, Taylor G. In a retrospective study of chronic obstructive pulmonary disease inpatients, respiratory comorbidities were significantly associated with prognosis J Clin Epidemiol. 2005;58(11):1199-1205. 
6. Restrepo MI, Mortensen EM, Pugh JA, Anzueto A. COPD is associated with increased mortality in patients with community-acquired pneumonia. Eur Respir J. 2006;28(2):346-351.

7. Molinos L, Clemente MG, Miranda B, et al; ASTURPAR Group. Community-acquired pneumonia in patients with and without chronic obstructive pulmonary disease. J Infect. 2009;58(6):417-424.

8. Dey AB, Nagarkar KM, Kumar V. Clinical presentation and predictors of outcome in adult patients with community-acquired pneumonia. Natl Med J India. 1997;10(4):169-172.

9. Fine MJ, Auble TE, Yealy DM, et al. A prediction rule to identify low-risk patients with community-acquired pneumonia. $N$ Engl J Med. 1997;336(4):243-250.

10. Torres A, Peetermans WE, Viegi G, Blasi F. Risk factors for communityacquired pneumonia in adults in Europe: a literature review. Thorax. 2013;68(11):1057-1065.

11. Waterer GW, Kessler LA, Wunderink RG. Medium-term survival after hospitalization with community-acquired pneumonia. Am J Respir Crit Care Med. 2004;169(8):910-914.

12. Corrales-Medina VF, Musher DM, Wells GA, Chirinos JA, Chen L, Fine MJ. Cardiac complications in patients with community-acquired pneumonia: incidence, timing, risk factors, and association with shortterm mortality. Circulation. 2012;125(6):773-781.

13. Global strategy for diagnosis, management, and prevention of COPD [Updated 2015]. The global initiative for chronic obstructive lung disease; 2015. Available from: http:/goldcopd.org/gold-reports/. Available from: http://www.goldcopd.it/materiale/2015/GOLD_Report_2015.pdf. Accessed June 16, 2016.

14. Ernst P, Gonzalez AV, Brassard P, Suissa S. Inhaled corticosteroid use in chronic obstructive pulmonary disease and the risk of hospitalization for pneumonia. Am J Respir Crit Care Med. 2007;176(2):162-166.

15. Drummond MB, Dasenbrook EC, Pitz MW, Murphy DJ, Fan E. Inhaled corticosteroids in patients with stable chronic obstructive pulmonary disease: a systematic review and meta-analysis. JAMA. 2008;300(20): 2407-2416.

16. Singh S, Amin AV, Loke YK. Long-term use of inhaled corticosteroids and the risk of pneumonia in chronic obstructive pulmonary disease: a meta-analysis. Arch Intern Med. 2009;169(3):219-229.

17. Cavallazzi R, Singh S. Inhaled corticosteroids in chronic obstructive pulmonary disease: how significant is the risk of pneumonia and should it impact use of inhaled corticosteroids? Curr Infect Dis Rep. 2011; 13(3):296-301.

18. Welte T. Inhaled corticosteroids in COPD and the risk of pneumonia. Lancet. 2009;374(9691):668-670.

19. Balbi B, Majori M, Bertacco S, et al. Inhaled corticosteroids in stable COPD patients: do they have effects on cells and molecular mediators of airway inflammation? Chest. 2000;117(6):1633-1637.

20. Fine JP, Gray RJ. A proportional hazards model for the subdistribution of a competing risk. J Am Stat Assoc. 1999;94(446):496-509.

21. Sibila O, Mortensen EM, Anzueto A, Laserna E, Restrepo MI. Prior cardiovascular disease increases long-term mortality in COPD patients with pneumonia. Eur Respir J. 2014;43(1):36-42.
22. Holter JC, Ueland T, Jenum PA, et al. Risk factors for long-term mortality after hospitalization for community-acquired pneumonia: a 5-year prospective follow-up Study. PloS One. 2016;11(2):e0148741.

23. Tsiligianni IG, Kosmas E, Van der Molen T, Tzanakis N. Managing comorbidity in COPD: a difficult task. Curr Drug Targets. 2013;14(2): 158-176.

24. Campo G, Pavasini R, Malagu M, et al. Chronic obstructive pulmonary disease and ischemic heart disease comorbidity: overview of mechanisms and clinical management. Cardiovasc Drugs Ther. 2015;29(2): 147-157.

25. Sidney S, Sorel M, Quesenberry CP Jr, DeLuise C, Lanes S, Eisner MD. COPD and incident cardiovascular disease hospitalizations and mortality: kaiser permanente medical care program. Chest. 2005;128(4): 2068-2075.

26. Macchia A, Monte S, Romero M, D'Ettorre A, Tognoni G. The prognostic influence of chronic obstructive pulmonary disease in patients hospitalised for chronic heart failure. Eur J Heart Fail. 2007;9(9): 942-948.

27. Kaplan V, Angus DC, Griffin MF, Clermont G, Scott Watson R, Linde-Zwirble WT. Hospitalized community-acquired pneumonia in the elderly: age- and sex-related patterns of care and outcome in the United States. Am J Respir Crit Care Med. 2002;165(6):766-772.

28. Mortensen EM, Kapoor WN, Chang CC, Fine MJ. Assessment of mortality after long-term follow-up of patients with communityacquired pneumonia. Clin Infect Dis. 2003;37(12):1617-1624.

29. Müllerova H, Chigbo C, Hagan GW, et al. The natural history of community-acquired pneumonia in COPD patients: a population database analysis. Respir Med. 2012;106(8):1124-1133.

30. Ishiguro T, Takayanagi N, Yamaguchi S, et al. Etiology and factors contributing to the severity and mortality of community-acquired pneumonia. Int Med. 2013;52(3):317-324.

31. Crim C, Calverley PM, Anderson JA, et al. Pneumonia risk in COPD patients receiving inhaled corticosteroids alone or in combination: TORCH study results. Eur Respir J. 2009;34(3):641-647.

32. Lange P, Vestbo J, Nyboe J. Risk factors for death and hospitalization from pneumonia. A prospective study of a general population. Eur Respir J. 1995;8(10):1694-1698.

33. Barnett K, Mercer SW, Norbury M, Watt G, Wyke S, Guthrie B. Epidemiology of multimorbidity and implications for health care, research, and medical education: a cross-sectional study. 2012;380(9836):37-43.

34. Ilavska S, Horvathova M, Szabova M, et al. Association between the human immune response and body mass index. Hum Immunol. 2012; 73(5):480-485.

35. Janson C, Larsson K, Lisspers KH, et al. Pneumonia and pneumonia related mortality in patients with COPD treated with fixed combinations of inhaled corticosteroid and long acting beta2 agonist: observational matched cohort study (PATHOS). BMJ. 2013;346:f3306.
International Journal of COPD

\section{Publish your work in this journal}

The International Journal of COPD is an international, peer-reviewed journal of therapeutics and pharmacology focusing on concise rapid reporting of clinical studies and reviews in COPD. Special focus is given to the pathophysiological processes underlying the disease, intervention programs, patient focused education, and self management protocols.
Dovepress

This journal is indexed on PubMed Central, MedLine and CAS. The manuscript management system is completely online and includes a very quick and fair peer-review system, which is all easy to use. Visit http://www.dovepress.com/testimonials.php to read real quotes from published authors. 